\title{
PREDICTING SUCCESS IN FOSTER PLACEMENT: The Contribution of Parent-Child Temperament Characteristics
}

\author{
Jenny L. Doelling, Ph.D., and James H. Johnson, Ph.D.
}

\begin{abstract}
Parent and child temperament variables were examined in an interactive "goodness of fit" model with respect to foster placement outcome. The "mismatch" of a rigid foster mother and a child of negative mood was found to be predictive of less successful placement outcome, as was the placement of a child of more negative mood than expected by the foster mother.
\end{abstract}

$\mathrm{F}$ oster care is widely used to provide both short-term and long-term care for children. While foster care is usually preferable to an abusive or neglectful home, or to institutional placement, it is not without its problems. Abuse and neglect by foster parents does occur, and the provision of less than adequate care in foster homes does not seem to be uncommon (Martin \& Beezley, 1976). Additionally, many foster placements break down soon after initial placement (Stone \& Stone, 1983). While more research is needed, several studies have suggested that difficulty experienced by the child in foster care is related to various qualities of the foster parents (Cautley \& Aldridge, 1975; Fanshel \& Shinn, 1978b; Martin \& Beezley, 1976; Murray, 1984; Rowe, 1976; Stone \& Stone, 1983; Wiehe, 1982), to specific child characteristics (Cautley \& Aldridge, 1975; Fanshel \& Shinn, 1978a; Murray, 1984; Rowe, 1976; Stone \& Stone, 1983 ), and to the number of foster placements already experienced by the child (Fanshel \& Shinn, 1978b; Murray, 1984; Pardeck, 1983; Stone \& Stone, 1983).
Several studies have provided preliminary information on foster parent and foster child characteristics thought to be predictive of placement success. Rowe (1976), for example, found that foster parents who are tolerant of child behavior that opposes their own values or wishes, such as poor academic performance, early adult behavior (e.g., smoking, drinking, sexual activity), difficult social behavior of the latency age child, or lack of strict religious observance are more successful as foster parents. Similarly, Cautley and Aldridge (1975) found that foster parents who could handle common problem behavior less harshly, without excessive discipline, and with understanding were more successful. They also found that ability to handle defiant and withdrawn behavior was predictive of success.

Foster children characteristics have also been found to relate to success of placement. Fanshel and Shinn (1978a) found that children whose behavior was characterized as defiant and hostile tended to experience more replacements. Stone and Stone (1983) found that less aggressive, better socialized

Submitted to the Journal in October 1989. The authors are at the Department of Clinical and Health Psychology, University of Florida, Gainesville. 
children were more likely to remain in their assigned placement.

In most research, foster parent and child variables have been examined independently. A case may be made, however, for assessing how the interactions of various foster parent and foster child characteristics relate to placement outcome. A parentchild interactive approach with potential relevance to foster placement outcome has been suggested by Thomas, Chess, and Birch (1968) and by Thomas and Chess (1977) in their consideration of the concept of temperament. Thomas et al. have conceived of childhood temperament along an easyto-difficult continuum on which difficult children are seen as being irregular in biological functioning (eating, sleeping, toileting), withdrawing from and adapting slowly to environmental changes, displaying intense emotional responses, and showing predominantly negative rather than positive moods. More recent conceptualizations have included high activity level, rigid responses, and distractibility (Lerner, Palermo, Spiro, \& Nesselroade, 1982). Easy children have the opposite pattern on each of these dimensions.

Rather than focusing exclusively on child characteristics, Thomas and Chess (1977) stressed the importance of the interaction or "goodness-of-fit" between child and parent and argued that the parental response to the child's temperament-related behavior mediates the relationship between temperament and the development of behavior problems in difficult children (Cameron, 1977). Just as child adjustment may relate to the goodness of fit between child temperament and the demands of the environment, these data suggest that the goodness of fit between foster parent and foster child temperaments may be related to successes of foster placement.

In a study of risk factors in abusive behavior, Johnson, Floyd, and Isleib (1986) found a "mismatch" between parent and child temperament to be highly predictive of abusive and neglectful behavior. Specif- ically, it was found that a particular type of mismatch - a child of difficult temperament paired with an unadaptive parentwas present in almost half of the abusive and neglectful families and was not present in any of the nonabusive families involved in the study. While Thomas and Chess (1977) considered goodness-of-fit as it relates to the development of later child psychopathology, a similar concept seems applicable to outcomes of child abuse and also, perhaps, to the failure of foster care placements.

Many of the parent and child variables assessed in previous foster care studies paralleled to some degree the dimensions associated with the concept of temperament.

However, most of these variables have not been found to be predictive of success or failure when considered individually. As it seems likely that a variation on the interactive, or goodness-of-fit, model may be a useful way of examining the success of foster care placements, the present study was designed to examine the extent to which a foster parent-foster child temperament mismatch might be predictive of foster placement outcome. Specifically, based on the earlier findings of Johnson et al. (1986), it was predicted that a mismatch between a difficult child and an unadaptive foster parent would be most highly correlated with poor outcome.

\section{METHOD}

\section{Subjects}

Subjects were recruited through the local district office of the Florida Department of Health and Rehabilitative Services (HRS) and included foster families from seven north Florida counties. Children with major impairments such as mental retardation and physical handicaps were excluded, as were children intentionally placed for a short, interim period.

The sample consisted of 51 foster children ( 27 boys and 24 girls) whose age range was from five to ten years with a mean age of 7 years. Their average length of place- 
ment at the time of data collection was eight months. The foster mothers ranged in age from 21 to 69 years (mean age, 48 years) and foster fathers' ages ranged from 23 to 74 (mean age, 59 years). On average, the families had either one or no natural or adopted children, and two or three other foster children living in the home. Some homes, however, had as many as three natural, four adopted, and five other foster children. Mean annual family income was $\$ 18,265$. The average education for both mothers and fathers was high school level. In 46 cases, the primary caretaker was the mother; in the remaining 5 , both parents were caretakers. Forty-three children had two parents, and eight children had a foster mother only.

\section{Measures}

A demographic questionnaire was completed by each foster mother. Information sought included child's age, parents' ages, length of the placement, number of natural, adopted, and other foster children in the home, income, educational level of parents, number of hours the foster child spent with each parent, number of parents in the home, and which parent was the primary caretaker.

The Dimensions of Temperament SurveyRevised (DOTS-R) is a measure developed by Lerner, Palermo, Spiro, and Nesselroade (1982) and revised by Windle and Lerner (1986). Both versions were designed to identify features of temperament continuous in the behavioral repertoire from early childhood through adulthood. Factor analyses of a 54-item revised version revealed a nine-factor model for the child versions and a ten-factor model for the adult version. Eight of the attributes are identical for both versions: Activity Level-General, Activity Level-During Sleep, ApproachWithdrawal, Flexibility-Rigidity, Mood, Rhythmicity-Sleep, Rhythmicity-Eating, and Rhythmicity-Daily Habits. The ninth attribute on the child versions is Task Orientation which differentiates into two at- tributes in the adult version: Distractibility and Persistence. Internal consistency coefficients (Cronbach's alpha) for these dimensions range from .54 to .91 . While additional research with the DOTS-R is needed, the available data support its use as a research measure for studies of child and adult temperament.

The DOTS-R ADULT was completed by each foster mother. The DOTS-R CHILD on each child was completed by both the foster mother and the child's teacher (the teachers were able to answer items for only five of the nine attributes, since they had no experience of the child's behavior in terms of the three rhythmicity dimensions or sleep activity level).

Additionally, a modified version of the DOTS-R CHILD described by Lerner et al. (1985) was used to assess foster mothers' general expectations of child temperament. For example, the DOTS-R item "My child smiles often" was changed to "I expect a child to smile often." This version of the DOTS-R was included as another way of assessing the goodness-of-fit between child temperament and environmental demands.

The Family Environment Scale (FES) (Moos \& Moos, 1981) is a 90 -item scale which measures the social-environmental characteristics of families. The Conflict subscale of the FES was targeted as a dependent measure relevant to success of placement. For the FES subscales, internal consistencies (Cronbach's alpha) range from .61 to .78 ; test-retest reliabilities (at a twomonth interval) range from .68 to .86 ; and normative data collected from 1,125 families include families from all areas of the country, from ethnic minority groups, from all age groups, and single-parent and multigenerational families.

The Foster Placement Evaluation Scale (FPES) is a 14-item scale developed by the authors for use in the present study. It was designed to measure success of foster placements as assessed by foster care case workers. In developing the scale, interviews were initially conducted with nine foster care 
workers in order to generate an item pool. A preliminary scale consisting of 16 items was then evaluated by these same workers as well as by ten clinical psychology graduate students and faculty to identify those related to placement success. All but two of the items were judged to be useful and no omission was mentioned consistently enough to be added to the scale. Fourteen items, on a scale of one to five, were thus retained for response by foster care workers.

It should be pointed out that items on this scale are highly reflective of dimensions discussed by Wolins (1963) as important in evaluating foster placements. The specific areas addressed by the scale include physical care, affection, acceptance of the child, equal treatment of the child and other children in the home, ability to get along with the child's natural parents, ability to deal with behavior problems, awareness of the child's individual needs, amount of time spent playing with the child, amount of time spent in general with the child, the child's academic performance and behavior in school, quality of the child's relationships with other children in the home, and the child's degree of adaptation to the family structure.

Measures of internal consistency indicate excellent reliability of the scale. The split-half correction coefficient (SpearmanBrown formula) for odd and even items was .90. The mean item-to-total correlation (Cronbach's alpha) was .88. Reliability as calculated on the 29 cases for which both a primary case worker and supervisor rating were available was also acceptable. The correlation coefficient between the two raters' scores was .65, indicating moderate agreement. This measure of reliability is believed to be lower due both to the reduced number of cases on which two ratings were available and to the fact that case workers and supervisors had different degrees of familiarity and different sources of information about the families. Previous investigators have found interrater reliabilities on pairs of foster care workers to be consistently low to moderate (Fanshel, 1961; Wolins, 1959, 1963). Given these findings and limitations, the obtained interrater reliability coefficient appears quite adequate and higher than that reported by Fanshel (1961). Overall reliability of the scale, therefore, appears good and supports the use of the measure in the present study.

A foster mother satisfaction rating was obtained as an additional measure of placement success. Each foster mother was asked to rate her degree of satisfaction with the placement on a five-point scale (very dissatisfied, somewhat dissatisfied, neutral, somewhat satisfied, very satisfied). While perhaps more subjective than case worker evaluations, these ratings were seen as a useful source of additional data on placement outcome.

\section{Procedure}

Informed consent was obtained via a written consent form. The foster parents completed the demographic questionnaire, the mother's satisfaction rating, a DOTS-R ADULT on themselves, the modified DOTS-R CHILD that assessed parents' expectations of child temperament, a DOTSR CHILD on the target child and the FES, in that order. Initial plans were for completion of each form by both parents. However, several homes had only one parent (mothers in every case) and, in many others, the foster father was either unavailable or unwilling to participate. It was therefore necessary to drop the father data (obtained in only 13 cases) from the analyses.

The children's teachers also completed a DOTS-R CHILD on the target child. In 21 cases, the child was either not yet in school, or the child's teacher was unavailable or unwilling to participate. Teacher data was collected on the remaining 30 cases.

Foster care case workers completed an FPES for each placement for which they were primarily responsible. Foster care supervisors were asked to identify those cases with which they had both independent con- 
tact and adequate familiarity, and to complete forms for those placements. This was possible in 29 of the cases. The primary case workers' ratings, which were available in all cases and deemed to be the more accurate ratings, were used in the analyses. The supervisors' ratings were used only in analyses assessing the correlations between the two ratings.

Initial data analyses involved an assessment of the direct relationship between measures of temperament and degree of successful placement. Subsequent analyses were designed to determine the joint contribution of parent and child temperament characteristics and the prediction of positive outcome.

\section{RESULTS}

\section{Dependent Measures}

In terms of data analyses, it was first necessary to examine the three dependent measures to determine whether they measured the same or different outcome attributes. The correlation between case worker ratings on the FPES and mothers' ratings of satisfaction approached significance $(r=.26, p=.06)$, suggesting that these measures are somewhat related but not identical. There was no significant correlation between the Conflict subscale scores on the FES and FPES scores $(r=-.17$, $p=.23)$ or mothers' satisfaction ratings $(r=$ $-.03, p=.83$ ). As each of these three variables appears to measure somewhat different attributes, all were considered in the analyses.

\section{Temperament and Placement}

Initial analyses were performed to assess the degree to which single temperament variables were related to success of placement as assessed by the FPES. Only one mother temperament dimension, Activity Level During Sleep, was negatively correlated $(r=$ $-.34, p<.05$ ) with FPES scores. While the importance of this finding is unclear, this dimension may be an indicator of restlessness or anxiety, suggesting that anxious foster mothers tend to have less successful placements. Similarly, only one dimension on the expectations form of the DOTS-R CHILD was positively correlated with FPES scores. Mothers who reported general expectation of high sleep-activity levels in children $(r=.41, p<.01)$ had more successful placements. It is possible that mothers with unrealistic expectations of children's restlessness tend to have less successful placements. No child temperament dimensions were significantly correlated with FPES scores, nor were any demographic variables. Considered alone, these variables thus appear to explain very little in terms of predicting success or failure of foster placements.

\section{Goodness-of-Fit and Placement}

A major hypothesis of the study was that a combination of parent-child variables would be most predictive of placement success. Therefore, analyses were designed to consider these variables simultaneously. A multiple regression analysis with interaction terms was considered but deemed inappropriate because of the abnormality and skewness of FPES scores, Conflict subscale scores, and mother satisfaction ratings. Chi-square analyses were used as an alternative way to focus on the relationship between mother-child temperament matches or mismatches and placement success or failure. Children were categorized as "easy" or "difficult" on each DOTS-R temperament dimension; mothers were similarly categorized on the Flexibility-rigidity and Approach-Withdrawal DOTS-R temperament dimensions. Placements were characterized as relative successes or failures, depending on whether they scored above or below the median on the FPES, Conflict subscale, and mother satisfaction rating. A mother-child temperament combination was labeled a "mismatch" if both mother and child were difficult on that pair of dimensions and was labeled a "match" if one or both were easy on that pair of dimensions. The FlexibilityRigidity and Approach-Withdrawal dimen- 
sions of the DOTS-R ADULT were chosen as the primary parent variables for consideration. This was because they bore the closest resemblance both to the concept of tolerance-intolerance shown by previous authors to relate to success of placement (Cautley \& Aldridge, 1975; Rowe, 1976; Wiehe, 1982), and to the adaptive-unadaptive temperament dimension found to be associated with child abuse (Johnson, Floyd, \& Isleib, 1986). While additional dimensions of maternal temperament were assessed, particular combinations of interest were isolated because analyses for all possible combinations of parent and child temperament characteristics would have been so many as to greatly increase the probability of Type I errors.

Several findings were significant. A mismatch of an inflexible mother with a negative mood child was found to be predictive of poorer placement outcomeusing FPES scores $\left(\chi^{2}=4.2, p<.05, \phi=-.29\right)$, mothers' satisfaction ratings $\left(\chi^{2}=10.5, p<.01\right.$ [Fisher's exact test], $\phi=-.45)$ and FES Conflict subscale scores $\left(\chi^{2}=4.2, p<.05\right.$, $\phi=-.29$ ).

Goodness-of-fit may also be assessed in terms of the difference between mothers' expectations of child temperament and the child's actual temperament characteristics. Difference scores between the two measures were therefore calculated for each temperament dimension. For any dimension, children whose temperament attributes met or exceeded (in a positive direction) mothers' expectations on that dimension were categorized as matches; children whose temperament attributes did not meet mothers' expectations (i.e., were more difficult) were categorized as mismatches. Using scores on the FPES as the dependent variable, a mismatch of a mother with a child of more negative mood than she expected was predictive of poorer placement outcome $\left(\chi^{2}=7.9, p<.01, \phi=.39\right)$. Thus, the mismatch of an inflexible mother with a child of negative mood was predictive of poorer placement outcome, as defined by all three measures. The mismatch of a mother with a child who did not meet her expectations for mood was also predictive of poorer placement outcome in terms of FPES scores. Conversely, matches were predictive of more successful placement.

A less relevant, yet significant, relationship was found between a mismatch of inflexible mothers with children displaying high sleep-activity levels and poorer placement outcome, using FPES scores as the dependent measure $\left(\chi^{2}=4.2, p<.05, \phi=\right.$ - .29). A significant relationship was also found between inflexible mothers mismatched with children of low sleep rhythmicity and lower mother satisfaction ratings $\left(\chi^{2}=9.8, p<.01, \phi=-.44\right)$, and between inflexible mothers mismatched with children of low eating rhythmicity and lower mother satisfaction ratings $\left(\chi^{2}=5.8, p<\right.$ $.05, \phi=-.34)$.*

Assessment of maternal characteristics of Approach-Withdrawal yielded no significant relationships.

\section{DISCUSSION}

These results support an interactive parent-child temperament approach to prediction of outcome in foster care placements. Individual variables related to mother and child temperament characteristics did not in themselves explain placement outcomes. However, when placements were examined in terms of goodness-of-fit between certain dimensions of mother and child temperament, a number of significant relationships were found. Specifically, the combination of an inflexible mother and a child with negative mood was shown to predict relative placement failure in terms of greater conflict, lower maternal satisfaction, and

\footnotetext{
* Reported $p$-values are those for Fisher's exact test: expected cell frequences were less than five for "failed" placements because very few of the mothers reported less than high satisfaction with the placements, and this was the one measure on which a median split was not possible.
} 
case workers' ratings of placement success. The present findings, in fact, suggest that this is the most troublesome combination in terms of placement outcome.

Mothers with children of more negative mood than they expected also had less successful placements, as rated by case workers. Less intuitively acceptable is the finding that the combination of an inflexible mother and a child of low-rhythmicity was predictive of poorer outcome in terms of mothers' satisfaction with the placement. Thus, it appears that mothers may find less predictable children to be more difficult, and may be less satisfied with them, but can still provide adequate care without great conflict.

The mechanism through which the relevant types of mismatch exert their influence is not clear, however. The finding of greater conflict in mismatched placements suggests that part of the problem lies in negative interaction patterns resulting from the mismatches. A most desirable study would be one in which the actual nature of family interaction patterns in matched versus mismatched placements was explored. This might be accomplished by including direct observation of family interactions so as to discover the channels which through a mismatch exerts its influence. While the present findings suggest that greater conflict occurs in these placements, it would be informative to define the nature of these interactions more precisely.

It is unfortunate that the foster fathers of the children in the study were for the most part inaccessible, since this made it difficult to assess the interaction of temperament between foster fathers and children. However, this was not seen as a major problem since the mother was the primary caretaker in most cases, while both parents were primary caretakers in only five. Thus, the relationship between mother and child was probably the crucial one in most cases in terms of placement outcome. Nevertheless, foster father data would be highly desirable in future studies.
A potential limitation of the study lies in the use of mothers' ratings of child temperament. These ratings of child temperament might be biased, and mothers might rate their foster children as uniformly more easy or difficult than they actually are, possibly as a result of the mothers' own temperament characteristics (e.g., rigid mothers might rate children as more difficult than would flexible mothers). For this reason, teacher ratings of child temperament were also collected in $\mathbf{3 0}$ cases for the five (of nine) dimensions that could be assessed by teachers. The correlations between mother and teacher ratings were .13 for FlexibilityRigidity, .26 for Mood, $.32(p<.10)$ for Task Orientation, $.37(p<.05)$ for Activity Level-General, and .42 $(p<.05)$ for Approach-Withdrawal (mean correlation = $.31, p<.10$ ). While indicative of only a low level of agreement, the correlations are consistent with those obtained in previous research (Bates, 1980; Field \& Greenberg, 1982).

However, in view of the substantial lack of agreement between mother and teacher ratings of child temperament, analyses were conducted to examine for consistent bias on the part of the mothers and for any effect such bias might have had on the results obtained. Mother and teacher ratings on each dimension were compared by means of $t$ tests. They indicated that mothers did not uniformly rate children as more difficult or more easy than did teachers. Furthermore, whatever differences existed were shown not to have affected the results. This lack of effect was assessed by subtracting the teacher rating from the mother rating for each subject on each dimension and thus obtaining a difference score. The difference scores of rigid and flexible mothers (categorized according to whether they fell above or below the median) were compared by $t$-tests and found not to be significantly different from each other on any of the five dimensions for which both mother and teacher ratings were available. It seems probable that the rather low correlation obtained 
between mother and teacher ratings is due to the reduced number of subjects on which both measures were available, to the fact that the DOTS-R CHILD is designed to be completed by parents rather than teachers, and to the teachers' limited opportunity to observe children on many of the relevant dimensions. Therefore, the mothers' ratings were chosen as more representative and accurate and without such bias as would influence the results. Most importantly, rigid and flexible mothers were not shown to have rated children significantly differently than did teachers.

Another limitation of the study arose from the choice of already existing placements (desirable because of their availability). This rendered the sample somewhat biased in favor of more successful placements because they had already lasted for several weeks, some for months, whereas previous research indicates that most breakdowns occur within four weeks of placement. (Stone \& Stone, 1983). This may help to explain the skewness of the dependent measures (i.e., more placements rated as successful). It is probable that more powerful results would have been obtained had more new placements (and thus potential breakdowns) been included in the sample. It is also impossible to know how many of the placements in the sample will eventually break down. However, at four- to sixmonth follow-up, eleven of the children included in the study had been moved to another foster home (some for reasons of poor outcome and some for unknown reasons); in six, an inflexible mother had been mismatched with negative mood child. The small number of cases prevented a statistical test of significance. However, it should be noted that $55 \%$ of the breakdowns were mismatches, while only $25 \%$ of the cases overall were so classified. Future research that examines cases as they are placed, thus maximizing the inclusion of the least successful placements (early breakdowns), would be desirable and likely to show an even more striking relationship between a mismatch of parent and child temperament and poor outcome of foster placements. A study such as this, or a similar one using other existing measures of temperament, would also be a means of replicating the present findings.

While the mismatch of inflexible mothers with negative mood children appeared in all of the analyses as predictive of poor placement outcome, this was not true in every individual case, suggesting the presence of other factors. It may make sense to think of this type of mismatch as a risk factor which might be either mediated or exacerbated by such other factors as marital discord, financial problems, or other stressors. These other factors were not addressed by this study and may also be a fruitful area for future research.

It is also debatable whether these types of mismatches should simply be avoided when making foster placements, or whether some specific parent training for flexibility and tolerance in parenting style might be effective in at-risk placements. Given careful replication of these findings and a delineation of the nature of the problematic interaction patterns within these families, research directed towards developing and assessing such training programs is suggested to explore this possibility.

\section{REFERENCES}

Bates, J.E. (1980). The concept of difficult temperament. Merrill-Palmer Quarterly, 26, 299-319.

Cameron, J.R. (1977). Parental treatment, children's temperament, and the risk of childhood behavioral problems: 1. Relationships between parental characteristics and changes in children's temperament over time. American Journal of Orthopsychiatry. 47, 568-576.

Cautley, P.W., \& Aldridge, M.J. (1975). Predicting success for new foster parents. Social Work, 20 , 48-53.

Fanshel, D. (1961). Studying the role performance of foster parents. Social Work, 6, 74-81.

Fanshel, D., \& Shinn, E.B. (1978a). Child behavior characteristics of foster children. In D. Fanshel \& E.B. Shinn (Eds.), Children in foster care: A longitudinal investigation (pp. 325-372). New York: Columbia University Press.

Fanshel, D., \& Shinn, E.B. (1978b). Discharge and other status outcomes. In D. Fanshel \& E.B. Shinn (Eds.), Children in foster care: A longitudinal in- 
vestigation (pp. 112-144). New York: Columbia University Press.

Field, T., \& Greenberg, R. (1982). Temperament ratings by parents and teachers of infants, toddlers and preschool children. Child Development, 53, 160-163.

Johnson, J.H., Floyd, B.J., \& Isleib, R. (1986). Parent, child and social variables as predictors of child abuse: Implications for a temperament mismatch view of abusive behavior. Unpublished manuscript, University of Florida, Gainesville.

Lemer, J.V., Lerner, R.M., \& Zabiski, S. (1985). Temperament and elementary school children's actual and rated academic performance: $A$ test of a "goodness-of-fit" model. Journal of Child Psychology and Psychiatry, 26, 125-136.

Lerner, R.M., Palermo, M., Spiro, A., \& Nesselroade, J.R. (1982). Assessing the dimensions of temperamental individuality across the life span: The Dimensions of Temperament Survey (DOTS). Child Development, 53, 149-159.

Martin, H.P., \& Breezley, P. (1976). Foster placement. In H.P. Martin (Ed.), The abused child (pp. 189-199). Cambridge, MA: Ballinger.

Moos, R.H., \& Moos, B.S. (1981). Family Environment Scale manual. Palo Alto, CA: Consulting Psychologists Press.

Murray, L. (1984). A review of selected foster careadoption research from 1978 to mid-1982. Child Welfare, 58, 113-124.
Pardeck, J.T. (1983). Marital status and family source of income: Potential predictors for determining the stability of foster family care? Adolescence, 18, 631635.

Rowe, D.C. (1976). Attitudes, social class, and the quality of foster care. Social Service Review, 50 , 506-514.

Stone, N.M., \& Stone, S.F. (1983). The prediction of successful foster placement. Social Casework, 64, 11-17.

Thomas, A., \& Chess, S. (1977). Temperament and development. New York: Brunner/Mazel.

Thomas, A., Chess, S., \& Birch, H. (1968). Temperament and behavior disorders in children. New York University Press.

Wiehe, V.R. (1982). Differential personality types of foster parents. Social Work Research \& Abstracts, 18, 16-20.

Windle, M., \& Lemer, R.M. (1986). Reassessing the dimensions of temperamental individuality across the life span: The Revised Dimensions of Temperament Survey (DOTS-R). Journal of Adolescent Research, 1, 213-230.

Wolins, M. (1959). The problem of choice in foster home finding. Social Work, 4, 40-48.

Wolins, M. (1963). Selecting foster parents: The ideal and the reality. New York: Columbia University Press.

For reprints: James H. Johnson, Ph.D., Dept. of Clinical and Health Psychology, Box J-165, JHMHC, University of Florida, Gainesville, FL 32610 